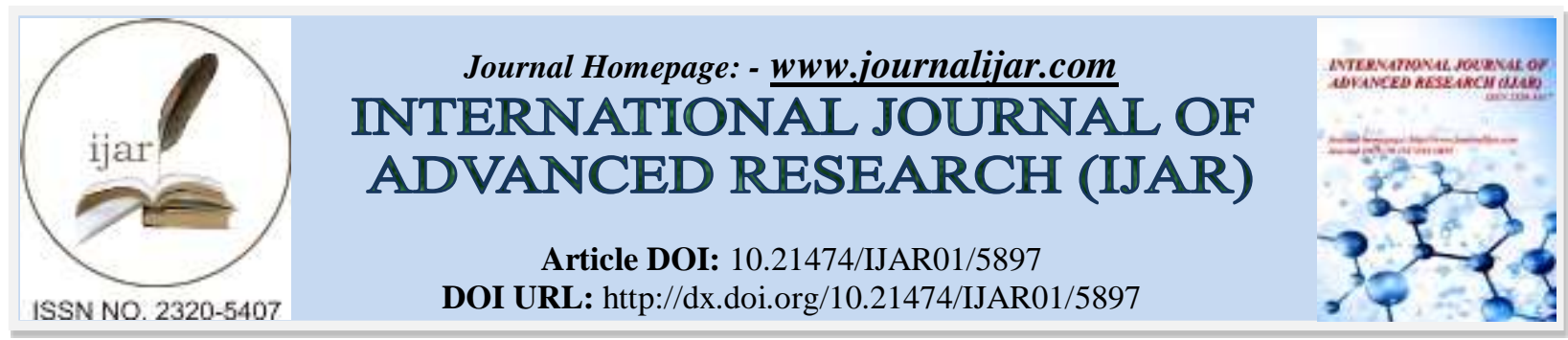

RESEARCH ARTICLE

\title{
THERAPEUTIC EFFECT OF P- COUMARIC ACID AGAINST SULFASALAZINE- INDUCED PULMONARY TOXICITY IN ADULT MALE RATS.
}

Aml Salem Saleh Ahmed.

Zoology Department, Women's College For Arts, Science And Education -Ain Shams University, Egypt.

\section{Manuscript Info}

Manuscript History

Received: 17 September 2017

Final Accepted: 19 October 2017

Published: November 2017

Key words:-

p- coumaric acid, sulfasalazine, lung injury, $\alpha$-SMA, collagen fibers.

\section{Abstract}

Objective: $p$ - Coumaric acid (p- CA) is an pervasive plant metabolite with antioxidant, anti- inflammatory and anticancer possessions. The present study was designed to estimate the curative effect of p- CA on sulfasalazine stimulated pulmonary injury.

Materials and Methods: 20 adult male albino rats were divided into 4 groups; group (1): control group received $0.5 \mathrm{ml}$ carboxy methyl cellulose (CMC), group (2): rats were treated with p- CA $(100 \mathrm{mg} / \mathrm{kg}$ b.w.), group (3): animals were treated with sulfasalazine $(600 \mathrm{mg} / \mathrm{kg}$ b.w.), (4): rats were treated with sulfasalazine associated with p-CA. The injection of both substances was orally for 2 weeks. At the end of the treatment, the rats were sacrificed and lungs were taken out for biochemical, histological, histochemical and immnuohistochemical examinations.

Results: Sulfasalazine caused pulmonary injury as judged by an increase in MDA tissue level and decrease in SOD and GSH levels. The thickness of alveolar epithelium and hemorrhage were observed. There was an increment in collagen fibers around bronchiole and in the lung interstitium. Moreover, positive alpha smooth muscle actin $(\alpha-$ SMA)- immunoreactivity detected within the cytoplasm of fibroblast cells. Administration of $\mathrm{p}$ - CA restorative these changes towards normal state.

Conclusion: The handling with p- CA could serve as a potential protective agent with therapeutic capabilities against sulfasalazine induced- lung toxicity.

Copy Right, IJAR, 2017,. All rights reserved.

\section{Introduction:-}

The lung is a highly particular and vigorous organ that has developed to exploit gas exchange. It is an object for a variety of possible toxic substances due to its large contact surface. It also may serve as a metabolism site for certain drugs (Schwaiblamir et al., 2012). Drug- induced lung damage may comprise each compartment of the lung. The most conventional form of drug- induced lung toxicity is drug- induced interstitial lung disease (DILD) (Schwaiblmair et al., 2012). Each compartment of the lung may be disturbed by chronic inflammation, with functional consequences that imitate the role of that structure. Patients suffer from rheumatoid arthritis have an increased sensitivity to chronic suppurative lung disease and bronchiolitis obliterans organizing pneumonia (BOOP) (Walsh and Pearson, 2001).

Corresponding Author:- Aml Salem Saleh Ahmed.

Address:- Zoology Department, Women's College For Arts, Science And Education -Ain Shams

University, Egypt. 
Sulfasalazine (sulfa) is a widely administrated drug against rheumatoid arthritis and other inflammatory- mediated disorders as Crohn's disease in the human (Corea, 2007). On the other hand, lung damage is one of the grave adverse incidents accompanied by sulfasalazine treatment. Some investigations implied the role of reactive oxygen species and oxidative stress in the organ injury induced by this drug (Linares et al., 2009 and Linares et al., 2011). Oxidative stress emerges to be one of the mechanisms of sulfasalazine- induced organ injury (Linares et al., 2011).

It could be expected that the administration of some antioxidants might be a therapeutic attitude. Hence, chemicals that acquire multifactorial defensive activity, including antioxidant properties may propose pharmacological benefit against sulfasalazine- induced lung injury (Heidari et al., 2016). There is a converse correlation between dietary phenolic compound intake and mortality from various diseases (Yoon et al., 2013). Phenolic compounds are a group of phenolic acids that are widely disseminated in whole grains, fruits, pears, vegetables and beverages as tea, coffee, wine and chocolate.

p- Coumaric acid (p- CA) [4- hydroxyphenyl-2- propenoic acid], a phenolic acid is a hydroxyl derivative of cinnamic acid. In plants, p- CA is a transitional product of the phenylpropanoid pathway, widely distributed in apples, pears, potatoes, pineapples, tomatoes, grapes, oranges, berries, onions, oats, wheat and beans. p- CA has been suggested to exhibit antioxidant, anti- inflammatory, anticancer activities (Lee et al., 2009; Kong et al., 2013 and Yoon et al., 2013). Also, it has neuroprotective, cardioprotective and anti- ulcer activities, respectively (Vauzour et al., 2010; Abdel- Wahab et al., 2003 and Barros et al., 2008).Keeping the above facts in view, the present study was designed to investigate the ameliorative effect of $\mathrm{p}$ - CA acid on sulfasalazine- induced lung lesion in rats.

\section{Materials and Methods:- Animals}

Twenty adult male albino rats weighing $(160 \pm 10 \mathrm{~g})$ were obtained from the Medical Research Centre Ain Shams University. Experimental protocols followed the Guidelines for the Care and Use of Laboratory Animals approved by the Institutional Ethics Committee of Ain Shams University. They were kept under observation for 2 weeks before the onset of the experiment to exclude any intercurrent infection. All the animals were kept in standardized cages (5 rats in each) at normal atmospheric temperature $\left(25 \pm 5^{\circ} \mathrm{C}\right)$, humidity $(55 \pm 5 \%)$ and with an alternating $12-\mathrm{h}$ light and dark cycle. During, the entire period of the study, the rats had access to food and water ad libitum.

\section{Chemicals:-}

Colosalazine- EC was purchased from Arab Caps, Egypt. p- Coumaric and carboxy methyl cellulose (CMC) were purchased from Sigma Chemical Company (St. Louis, MO, USA).

\section{Experimental design:-}

Group (1):(control) rats received $0.5 \mathrm{ml}$ of (CMC, $0.5 \%)$.

Group (2): rats were treated with p- CA (100mg/ kg b.w.).

Group (3): rats were treated with therapeutic dose of sulfa (600mg/ kg b.w.).

Group (4): rats treated with sulfa accompanied with p- CA.

All treatments were dissolved in $0.5 \%$ of carboxy methyl cellulose (CMC) and given daily for 2 weeks by gastric intubation. Furthermore, body weight of experimental groups was recorded and mean final body weight was calculated.

At the end of the treatment period, all rats were sacrificed. The lung was dissected out, washed in ice- cold saline and patted dry. The lung weight of each rat was recorded at the terminal sacrifice and the relative lung weight was calculated. A part of lung tissue was homogenized and 10\% tissue homogenate was used for estimation of various biochemical parameters. While, the other part of the lung was fixed in $10 \%$ buffered formalin solution and processed for paraffin sections of 5-7 $\mu \mathrm{m}$ thickness for histological studies.

\section{Biochemical estimations:-}

Determination of MDA, SOD and GSH levels according to the method of (Ohkawa et al. 1979; Nishikimi et al. 1972 and Beutler et al. 1963), respectively. 


\section{Histological\& histochemical studies:-}

Lung sections were stained with hematoxylin and eosin (H\& E) (Harris, 1900) and Masson's trichrome (Bancroft and Gamble, 2002).

Immunohistochemical study:-

Alpha smooth muscle actin ( $\alpha$ - SMA) immunohistochemical staining was done according to (Bancroft and Cook, 1994).

Statistical analysis:-

The data were expressed as mean \pm standard error (S.E). The results were analyzed using an analysis of variance one- way test (ANOVA) using SPSS 17 software. Statistically significant when the P value was $<0.05$ and highly significant when the $\mathrm{P}$ value $<0.001$ (Dawson and Trapp, 2001).

\section{Results:-}

Mean final body weights of control and treated rats are presented in (Table 1). At the end of the experiment, group (3) treated with therapeutic dose of sulfa exhibited a marked decrease $(p<0.001)$ of the body weight as compared to the control group. Group (4) treated with $\mathrm{p}$ - CA showed a significant $(\mathrm{p}<0.01)$ protection against body weight loss as compared to group (3).

Treatment with sulfa showed a substantial increase in the mean and relative lung weights, when they compared to control group. While the group treated with sulfa accompanied with p- CA displayed an obvious decrease for both mean and relative lung weights as tabulated in (Table 1).

The administration of sulfa created a prominent impairment of lung functions as the elevated MDA level (Table 1). Moreover, the pulmonary level of both SOD and GSH were significantly decreased. It was found that p- CA administration produced an expressive decrease in MDA level. While it caused an indicative increase in both SOD and GSH levels as presented in Table (1).

On examination, pulmonary tissue in normal control group bared both the bronchioles with normal epithelium and alveolar sac (Figs.1A,B\&C). Group (3) exposed degeneration in the bronchiolar epithelium, thickening of interalveolar septa, dilated congested a branch of pulmonary artery and accumulation of inflammatory cells within the interalveolar and the bronchiole (Figs.1D, E\&F). While in group (4), nearly normal pulmonary architecture with a slight damage in the bronchiole and alveolar wall was observed (Figs. 1G\&H).

Lung tissue of control group showed normal distribution of collagen fibers in the lung parenchyma as around the bronchiole and intra- alveolar septa (Fig.2A). In group (3), lung sections exhibited an extensive collagen fibers deposition in the thick in the intra- alveolar septa as well as around the pulmonary bronchiole and blood vessels (Figs.2B). Group (4) showed a few to moderate collagen fibers in the lung interstitium and around the bronchiole (Figs.2C).

Control group presented a deficiency of $\alpha$ - SMA immunoreactivity within the lining cells of the alveoli (Fig.3A). Group (3) displayed a positive $\alpha$ - SMA immunoreactivity detected within the cytoplasm of fibroblast cells (Fig.3B). While, the group (4) demonstrated pale $\alpha$ - SMA immunoreactivity within the cytoplasm of pneumocyte II cells (Fig.3C).

\section{Discussion:-}

Sulfasalazine is used as antimicrobial agent; however, its use is often allied with grave pulmonary complications, the most common of which is chronic interstitial pneumonitis with organizing pneumonia and pulmonary fibrosis (Bhullar et al., 2007).

Our findings of the body and lung weights confirm that sulfa administration caused a loss of body weight due to the loss of appetite and also owing to protein degradation as a result of oxidative damage process. Also, the increment of the lung weight might be due to the lung hypertrophy and the proliferation of pneumocytes type II (Durmus et al., 2005). 
The present work showed that the administration of sulfa stimulated free radicals generation and lipid peroxidation. These radicals cause severe destruction to the plasma membrane and interfere with cytoskeleton assembly and cause protein degradation (Linares et al., 2009 and Linares et al., 2011).

Moreover, lung tissue is particularly susceptible to the free radical injury because of the low level of free radical detoxifying agents as SOD and GSH. Sulfa administration induced oxidative stress in lung tissue by the alteration observed in the antioxidant defense system, both enzymatic and non- enzymatic (Linares et al., 2011).

The association between elevated pulmonary content of MDA and lowered pulmonary contents of SOD and GSH found in the study strongly proves the oxidative damage caused by sulfa. This observation has been supported by the findings of Serrano- Mollar et al. (2003); Linnebur and Parnes (2004) and Koulaouzidis et al. (2007).

The treatment with p-CA showed a significant decrease in MDA level and an increase in the levels of SOD and GSH. This may be due to the increase in GSH biosynthesis or reduce the oxidative stress leading to less degradation of both GSH and SOD (Shairibha and Rajaduarai, 2014).

In this study, examination of the pulmonary tissue of sulfa treated group showed severe alveolar damage in the form of heavy infiltration of thickened interalveolar septum with inflammatory cells associated with congested blood capillaries. Also, there was a proliferation of pneumocytes type II.

Proliferation of pneumocytes type II form predominant lining cells of the alveoli was consistent with the previous experimental studies submitted that pneumocytes type II might constitute the reserve epithelial cells of the alveoli, and its proliferation and hyperplasia were regarded as a sign of pneumocytes type I repair and manifested its underlying injury (Durmus et al., 2005).

The presence of congested blood vessels and cellular infiltration indicates the presence of inflammatory reactions that might be attributed to the release of IL-6 and IL-8 from a human lung epithelial cell line as a response to oxidative stress (Massey et al., 1995). Also, that might increase capillary permeability evoking an inflammatory response via activation of oxidative stress- sensitive signaling pathway (Zidan, 2010 and Abdel- Gawad et al., 2012).

This study showed an emphysema and alveolar wall destruction that might be explained by the increased interstitial collagen fiber deposition and marked cellular infiltration (Stankiewicz et al., 2002; Zidan, 2010 and AbdelGawad et al., 2012).

While the destruction of alveolar wall might be due to the excess of the protease or elastase activity unopposed by appropriate antiprotease regulation with subsequent elastic tissue destruction and ruptured septal walls. Also, matrix metalloproteinases derived from macrophages, neutrophils and toxic- free radicals that had a role in tissue destruction (Kumar et al., 2004 and Abdel- Gawad et al., 2012).

Confirming results of pulmonary sections stained with H\&E and Masson's trichrome stains. Sulfasalazine administration revealed a significant increase of $\alpha$ - SMA immunoreactive myofibroblast (a key marker for myofibroblast) as compared to the control group that may be explained by the ability of sulfasalazine to increase the number of the myofibroblasts (Kulkarni et al., 2013 and Sabry et al., 2014).

Concomitant administration of p- coumaric acid with sulfasalazine for 2 weeks showed a considerable protection of the pulmonary tissue. The pulmonary architecture was considerably conserved; the alveoli were distended and the intralveolar septa were mildly thickened because of the presence of some collagen fibers. Also, there was a mild cellular infiltration according to its antioxidant and antiinflamatory effects as previous reported (Yoon $\boldsymbol{e t}$ al., 2013 and Shairibha and Rajaduarai, 2014). 


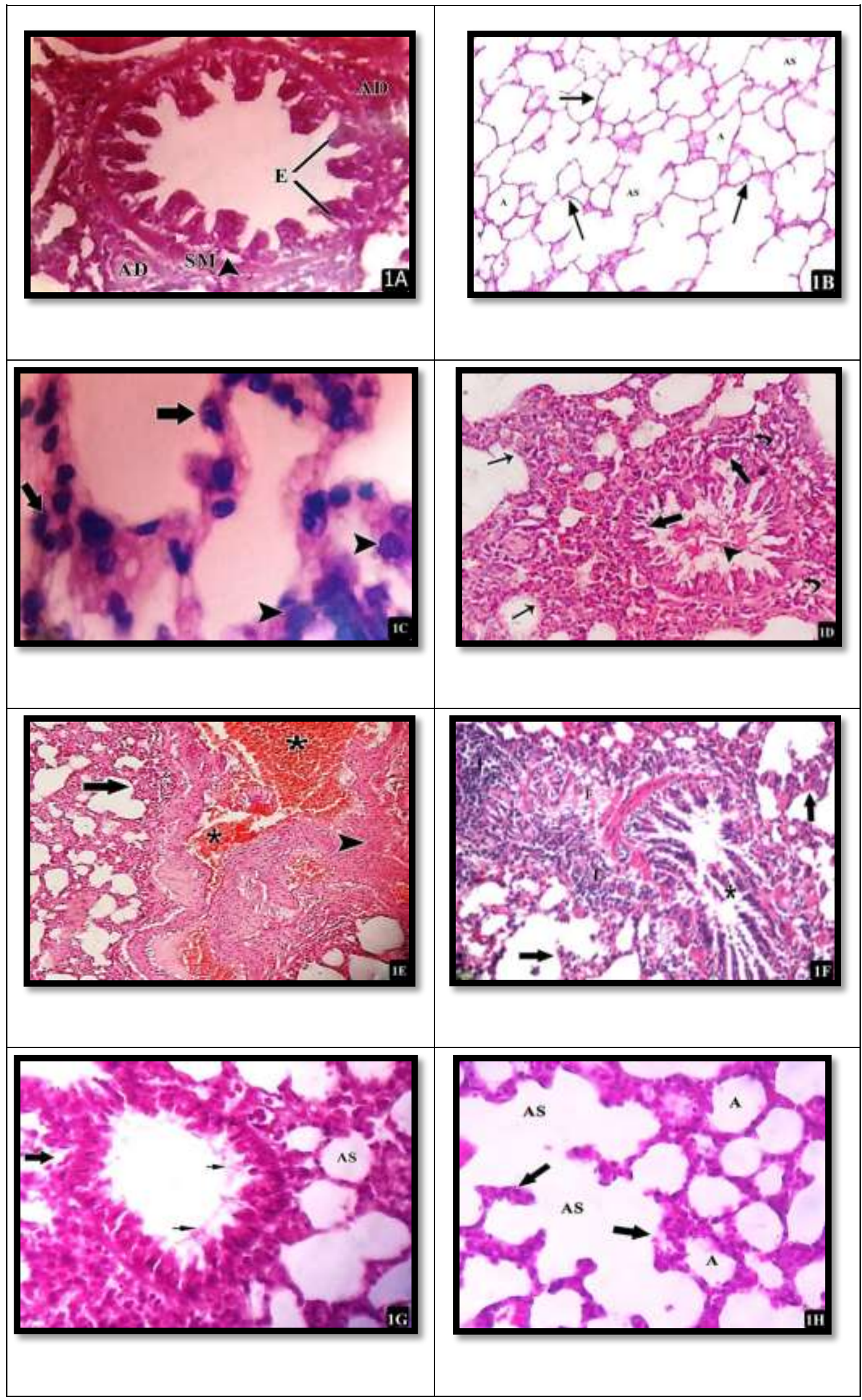


Figure 1:- embraces lung sections stained with H\&E (X 400). (A) control group showing lining epithelium (E), smooth muscle (SM), adventitia (AD) and lamina propria (arrow head). (B) showing alveolar sacs (AS), alveoli (A) with thin interalveolar septa (arrows). (C) showing type I pneumocytes (arrows) \& type II pneumocytes (arrow heads) in the alveolar wall. (D) sulfa treated group showing a marked degenerative changes in the bronchiolar epithelium (thick arrows), eosinophilic amorphous materials\& some of cell debris (arrow head) in the bronchiolar lumen, destructive basal lamina (curved arrows) and thickening of interalveolar septa (thin arrows) were observed. (E, X200) low magnification of lung section showing highly congested branch of pulmonary artery (*) thickening wall of this artery (arrow head) and thickening of interalveolar septum (arrow). (F) showing accumulation of inflammatory cells within the interalveolar septa (I), thickened wall of alveolar septa (arrows), degenerated lining epithelium of the bronchiole (*) and fibrotic area were also observed. (G) lung section of rat treated with p-CA associated with sulfa showing nearly normal pulmonary architecture with slight destructive bronchiolar basal lamina (thick arrow) and epithelail lining (short arrows). (H) showing trivial thickening of alveolar wall (arrows).

Table (1):- Mean final Body weight, lung and relative lung weights, oxidative and antioxidative parameters in control and treated groups (Mean \pm S.E.).

\begin{tabular}{|c|c|c|c|c|}
\hline $\begin{array}{l}\text { Groups } \\
\text { Parameters }\end{array}$ & $\begin{array}{l}\text { Group (1) } \\
\text { (Cont) }\end{array}$ & $\begin{array}{l}\text { Group (2) } \\
\text { (p- CA) }\end{array}$ & $\begin{array}{l}\text { Group } \\
\text { (Sulfa) }\end{array}$ & $\begin{array}{l}\text { Group (4) } \\
\text { Sulfa+ p- CA }\end{array}$ \\
\hline Mean final body weight (g) & $180.2 \pm 1.1$ & $177.5 \pm 1.1$ & $119 \pm 1.5_{3}^{\mathrm{a}}$ & $155.7 \pm 1.3_{2}^{b}$ \\
\hline Lung weight (g) & $0.537 \pm 0.36$ & $0.554 \pm 0.29$ & $1.11 \pm 0.55_{3}^{a}$ & $0.676 \pm 0.22^{b}{ }_{3}$ \\
\hline Relative lung weight (g) & $0.376 \pm 0.02$ & $0.369 \pm 0.02$ & $0.907 \pm 0.04_{3}^{a}$ & $0.507 \pm 0.01_{2}^{b}$ \\
\hline MDA (nmol/g tissue) & $0.16 \pm 0.05$ & $0.20 \pm 0.04$ & $2.2 \pm 0.21_{3}^{\mathrm{a}}$ & $0.7 \pm 0.08^{b}$ \\
\hline SOD $(\mathrm{U} / \mathrm{mg}$ tissue $)$ & $31.99 \pm 1.4$ & $31.07 \pm 2.6$ & $4.35 \pm 0.99^{\mathrm{a}}{ }_{3}$ & $25.57 \pm 1.6_{3}^{b}$ \\
\hline GSH (nmol/g tissue) & $33.69 \pm 0.97$ & $30.58 \pm 1.3$ & $5.56 \pm 0.67_{3}^{a}$ & $24.26 \pm 1.3_{3}^{b}$ \\
\hline
\end{tabular}

a: significant value compared to the control group.

b: significant value compared to sulfa group (therapeutic dose).

1: significant value at level $(\mathrm{p}<0.05) \quad 2$ : significant value at level $(\mathrm{p}<0.01)$

3 : significant value at level $(\mathrm{p}<0.001)$

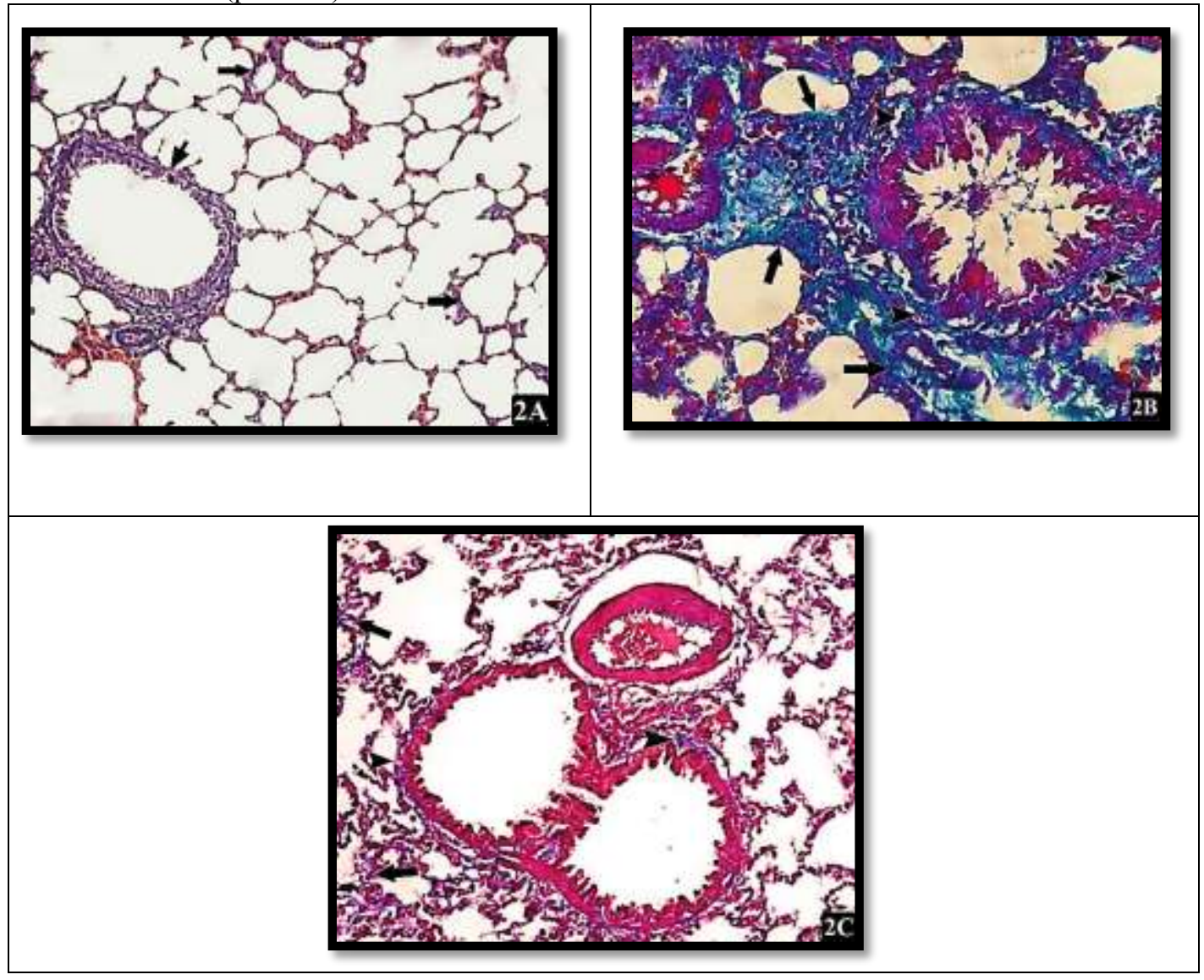


Figure 2:- includes sections in lung stained with masson trichrome. (A, X200) control section showing fine collagen fibers within the adventitia and lung interstitium. (B, X400) section in lung of sulfa treated group showing extensive collagen fibers deposition around the bronchiole (arrow heads) and in the lung interstitium (arrows). (C, X400) sulfa associated p- CA group showing faint to moderate collagen fibers deposition in the lung interstitium (arrows) and around the bronchiole (arrowheads).

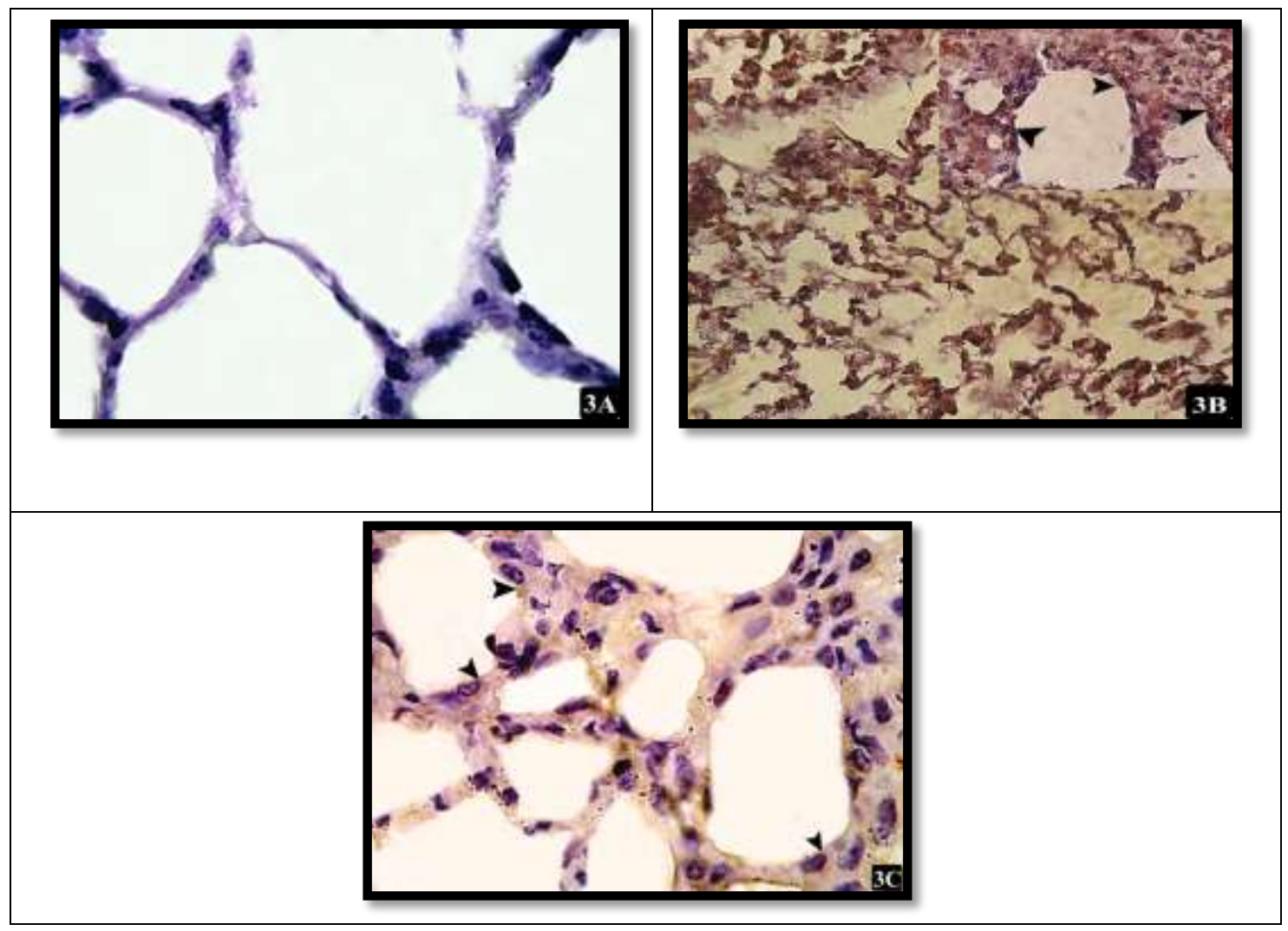

Figure 3:- contains sections in lung stained with $\alpha$ - SMA. (A, X400) control section showing lack of $\alpha$ - SMA immunoreactivity within the lining cells of the alveoli. (B, X200\&400) section in lung of sulfa treated group showing positive $\alpha$-SMA immunoreactivity within the cytoplasm of fibroblast cells (arrow heads). (C, X400) sulfa associated p- CA group showing faint $\alpha$-SMA immunoreactivity within the cytoplasm of pneumocyte type II cells

\section{References:-}

(arrowheads).

1. Abdel-Gawad, FA.,et al. (2014): Lung damage after long-term exposure of adult rats to sodium fluoride. Arch Med Sci., 10(5):1035-1040.

2. Abdel- Wahab, MH., et al. (2003): Influence of p- Coumaric acid on doxorubicin- induced oxidative stress in rat's heart. Pharmacol. Res., 48: 461- 465.

3. Bancroft, JD. and Cook, HC. (1994): Immunohistochemistry. In: Bancroft, JD. and Cook, HC.: Manual of Histological Techniques and their Diagnostic Applications. $2^{\text {nd }}$ ed. London, Madrid, Melbourne, New York, Tokyo: Churchill Livingstone, Edinburgh, 263- 325.

4. Bancroft, JD. and Gamble, M. (2002): Theory and Practice of Histological Techniques. $5^{\text {th }}$ ed., Churchill Livingstone, London, 167- 170.

5. Barros, MP., et al. (2008): Evaluation of antiulcer activity of the main phenolic acids found in Brazilian Green Propolis. J. Ethnopharmacol., 120 (3): 372- 377.

6. Beutler, E., et al. (1963): Colorimetric method for determination of glutathione reduced. J. Lab Clin. Med., 61: 882.

7. Bhullar, S., et al. (2007): Severe nitrofurantoin lung disease resolving without the use of steroids. J. Postgrad. Med., 53: 111- 113. 
8. Corea, N. (2007): Sulfasalazine. In: Enna, SJ.; Bylund, DB., eds. x Pharm: The Comprehensive Pharmacology Ref. New York: Elsevier: 1- 5.

9. Dawson, B. and Trapp, RG. (2001):. Basic and Clinical Biostatistics. $3^{\text {rd }}$ ed. New York, NY: Lang Medical Books/ McGraw- Hill.

10. Durmuş-Altun,G., et al. (2005): Use of iodine-123 metaiodobenzylguanidine scintigraphy for the detection of amiodarone induced pulmonary toxicity in a rabbit model: A comparative study with technetium- $99 \mathrm{~m}$ diethyltriaminepenta acetic acid radioaerosol scintigraphy, Ann. Nucl. Med., 19: 217- 224.

11. Harris, H.F. (1900): After Bruce Casselman W.C. (1959): Histochemical Technique, by Methuen and Co. LTd.

12. Heidari, R., et al. (2016): Sulfasalazine- induced renal and hepatic injury in rats and the protective role of taurine. Biolmpacts, 6 (1):3- 8.

13. Kong, CS., et al. (2013): Antiangiogenic effects of p- Coumaric acid in human endothelial cells. Phytother. Res., 27: 317- 323.

14. Koulaouzidis, A., et al. (2007): Nitrofurantoin-induced lung- and hepatotoxicity. Ann. Hepatol., 6(2):119-21.

15. Kulkarni, AA., et al. (2013): The triterpenoid CDDO-Me inhibits bleomycin-induced lung inflammation and fibrosis. PLoS One., 8(5):e63798.

16. Kumar, M., et al.(2004): Cellular and interactions in pulmonary fibrosis. Robbins and Cotran: Pathological Basis of Disease, $7^{\text {th }}$ ed. Library of Congress, Philadelphia, 27- 118.

17. Lee, SJ., et al. (2009): Evidence for the association of peroxidases with the antioxidant effect of p- Coumaric acid in endothelial cells exposed to high glucose plus arachidonic acid. BMB Reports, 42: 561- 567.

18. Linares, V., et al.(2009): Lipid peroxidation and antioxidant status in kidney and liver of rats treated with sulfasalazine. Toxicol., 256:152- 156.

19. Linares, V., et al.(2011): Oxidative stress as a mechanism underlying sulfasalazine- induced toxicity. Expert Opioion on Drug Safety, 10: 253- 263.

20. Linnebur, SA. and Parnes, BL. (2004): Pulmonary and hepatic toxicity due to nitrofurantoin and fluconazole treatment. Ann Pharmacother.,38(4):612-616.

21. Massey, TE., et al. (1995): Mechanisms in the pathogenesis of amiodarone-induced pulmonary toxicity. Can. J. Physiol. Pharmacol.,73(12):1675-1685.

22. Nishikimi, M., et al. (1972): The occurrence of superoxide anion in the reaction of reduced phenazine methosulphate and molecular oxygen. Biochem.Biophys. Res. Comm., 46(2), 849-854.

23. Ohkawa, H., et al. (1979): Assay for lipid peroxidation in animal tissues by thiobarbituric acid reaction. Annals of Biochemistry, 95, 351- 358.

24. Sabry, MM., et al. (2014): Histolgical and immunohistochemical study on the effect of stem cell therapy on bleomycin induced pulmonary fibrosis in albino rat. Int. J Stem Cells.,7(1):33-42.

25. Schwaiblmair, M., et al. (2012): Drug induced interstitial lung disease. The Open Respiratory Medicine J., 6: 63- 74.

26. Serrano-Mollar, A., et al. (2003): In vivo antioxidant treatment protects against bleomycin-induced lung damage in rats. Br J Pharmacol.,138(6):1037-1048.

27. Shairibha, R.S.M. and Rajadurai, M. (2014): Anti-diabetic effect of p-coumaric acid on lipid peroxidation, antioxidant status and histopathological examinations in streptozotocin-induced diabetic rats. Int. J. Int sci. Inn. Tech. Sec. B, 3:01-11.

28. Stankiewicz, A., et al. (2002): Effect of amifostine on lung oxidative stress after cyclophosphamide therapy. Bull. Vet. Inst. Pulawy., 46, 87-94.

29. Vauzour, D., et al. (2010): Caffeic acid, tyrosol and p- Coumaric acid are potent inhibitors of 5- S- Cysteinyldopamine induced neurotoxicity. Arch. Biochem. Biophys., 501: 106- 111.

30. Walsh, DA. and Pearson, CI. (2001): Angiogenesis in the pathogenesis of inflammatory joint and lung disease. Arthritis Res., 3 (3): 147- 153.

31. Yoon, SA., et al. (2013): p- Coumaric acid modulates glucose and lipid metabolism via AMP- activated protein kinase in L6 skeletal muscle cells. Biochem. And Bioohys. Res. Commun., 432: 553- 557.

32. Zidan, RA. (2010): Effect of long-term administration of amiodarone on rat lung and the possible protective role of vitamin E: A histological and immunohistochemical study. Egypt. J. Histol., 34: 117- 128. 